\title{
Dissipation in Langevin Equation and Construction of Mobility Tensor from Dissipative Heat Flow
}

\author{
Takashi UnEYAmA ${ }^{\dagger}$ \\ JST-PRESTO, and Center for Computational Science, Graduate School of Engineering, Nagoya University, \\ Furo-cho, Chikusa, Nagoya 464-8603, Japan \\ (Received: November 4, 2019)
}

\begin{abstract}
The rheological behavior of a material is strongly related to the energy dissipation, and the understanding and modeling of dissipation are important from the view point of rheology. To study rheological properties with some mesoscopic and macroscopic dynamics models, the modeling of dynamic equation which appropriately incorporates the dissipation is important. Although there are several methods to construct mesoscopic and macroscopic dynamic equations, such as the Onsager's method, their validity is not fully clear. In this work, we theoretically analyze the dissipation in a mesoscopic Langevin equation in detail, from the view point of stochastic energetics. We show that the dissipative heat flow from the heat bath to the system plays an important role in the mesoscopic dynamics. The dissipative heat flow is unchanged under the variable transform, and thus it is a covariant quantity. We show that we can construct the Langevin equation and also perform a coarse-graining based on the dissipative heat flow. We can derive the mobility tensor from the dissipative heat flow, and construct the Langevin equation by combining it with the free energy. Our method can be applied to various systems, such as the dumbbell model and the diffusion type equation for the density field, to give the coarse-grained dynamic equations for them.
\end{abstract}

Key Words: Langevin equation / Energy dissipation / Mobility / Coarse-graining

\section{INTRODUCTION}

To study the rheological behavior of some molecular and constitutive models, the energy dissipation plays an important role. When we deform a viscoelastic material, the material dissipates a part of the applied work into the heat. The simple models such as the Maxwell and Voigt models have viscous components (dash pots) which dissipate the energy. In the linear viscoelasticity, the loss modulus $G^{\prime \prime}(\omega)$ can be related to the energy dissipation under a small shear deformation at the angular frequency $\omega .{ }^{1)}$ Without the dissipation, we observe no viscous behavior and thus the system becomes totally elastic. Therefore, the understanding on the dissipation and the modeling which properly incorporates the dissipation are important. Also, the dissipation behavior depends on the time and length scales. At the microscopic scale, we have basically no dissipation. At the mesoscopic and macroscopic scales, we observe dissipation but the observed dissipation behavior depends on the scale (at least apparently). As the scale increases, generally the contribution of the dissipation increases. This is because the definition of the heat depends on the scale. ${ }^{2)}$

To describe the dynamics in the mesoscopic systems, the Langevin type equations ${ }^{3-5)}$ are widely employed. The

$\dagger$ Corresponding author.

E-mail: uneyama@mp.pse.nagoya-u.ac.jp

Tel: +81-52-788-6217
Langevin equation naturally incorporates the dissipation as the frictional force. In the macroscopic systems, phenomenological relaxation equations such as the time-dependent GinzburgLandau (TDGL) equation are useful. ${ }^{6)}$ The TDGL equation (without the noise term) is purely dissipative and the free energy of the system monotonically decreases as the time evolves, which is a common property of relaxation processes. The GENERIC (general equation for the nonequilibrium reversible-irreversible coupling) formalism ${ }^{7,8)}$ will be useful when there are both reversible and dissipative contributions in the system. These equations are, however, not fully trivial from the view point of fundamental statistical mechanics.

At the microscopic (or atomistic) scale, the system simply obeys classical mechanics. The equations of motion can be expressed in several different ways. A simple way is to employ the Newton's equation of motion. However, the Lagrange's dynamic equation and Hamilton's canonical equation in the analytic mechanics are much more general and useful, although they may not be intuitive. The Hamilton's principle states that in a mechanical system, a path $\boldsymbol{q}(t)$ which minimizes the action functional $\mathcal{S}[\boldsymbol{q}]$ is realized:9,10)

$$
\frac{\delta \mathcal{S}[\boldsymbol{q}]}{\delta \boldsymbol{q}(t)}=0,
$$


where $\delta / \delta \boldsymbol{q}(t)$ represents the functional derivative with respect to $\boldsymbol{q}(t)$. In this work, we call the condition in which the functional derivative becomes zero as the variational principle, since it is expressed in terms of the variation with respect to the path. The Hamilton's principle and the Fermat's principle are variational principles. ${ }^{10)}$ The action functional is expressed as the integral of the Lagrangian:

$$
\mathcal{S}[\boldsymbol{q}]=\int d t \mathcal{L}(\boldsymbol{q}(t), d \boldsymbol{q}(t) / d t)
$$

where $\mathcal{L}$ is the Lagrangian and is a function of $\boldsymbol{q}(t)$ and $d \boldsymbol{q}(t) / d t$. Eqs (1) and (2) give the Euler-Lagrange equation and thus the Langrange's dynamic equation:

$$
\frac{\partial \mathcal{L}(\boldsymbol{q}(t), d \boldsymbol{q}(t) / d t)}{\partial \boldsymbol{q}(t)}-\frac{d}{d t} \frac{\partial \mathcal{L}(\boldsymbol{q}(t), d \boldsymbol{q}(t) / d t)}{\partial(d \boldsymbol{q}(t) / d t)}=0 .
$$

The Hamilton's principle (1) holds even if we transform variable $\boldsymbol{q}(t)$ to another variable $\boldsymbol{q}^{\prime}(t)$. In this work, we may call a physical quantity or an equation is in a covariant form if it does not change its form under the variable transform. (Although the covariant property may look rather trivial, it is not always satisfied. For example, as we will discuss later, the free energy is not covariant.)

The Hamilton's principle (1) is powerful and useful because it is variational and covariant. However, we should notice that it can be usually applied only to systems without any dissipation. As we mentioned, for rheological properties at the mesoscopic and macroscopic systems, the dissipation becomes essential. Thus we should incorporate the dissipation into the dynamics, for example, by introducing the frictional force. For such purposes, we usually employ the Langevin equations or phenomenological TDGL type dynamic equations. It is generally not clear whether the variational and covariant properties are satisfied in these equations. A general method which is variational and/or covariant is desired, if it exists.

Many studies have been conducted to obtain a variational principle for dissipative systems which corresponds to the Hamilton's principle in the analytic mechanics. ${ }^{11-22)}$ Rayleigh $^{11,12)}$ proposed to use so-called the dissipation function to describe Lagrange's dynamic equations with nonpotential, friction force terms. Onsager ${ }^{23}$,24) also employed Rayleigh's dissipation function to describe the relaxation process in general linear nonequilibrium systems. However, both Rayleigh's and Onsager's methods are not in a variational form (a functional derivative form). Their dynamic equations do not have corresponding action functionals. As we will show later, their methods should be interpreted rather as the minimization condition.

We can, nonetheless, construct the action for such dissipative systems. Bateman ${ }^{13)}$ proposed to couple two systems to construct the action functional. In the Bateman's method, the dissipative system in which the total energy monotonically decreases, is coupled to the dual system in which the total energy monotonically increases. As a result, we obtain two set of dynamic equations and one of them is for the dissipative system. Bateman ${ }^{13)}$ also proposed to use an explicitly timedependent Lagrangian, as a variant of the coupled systems method. However, these methods are not physically natural. Riewe ${ }^{14)}$ proposed to employ the fractional derivatives to express the dissipative contributions. Although the Lagrangian with the fractional derivatives is a direct generalization of that in the analytic mechanics, it is questionable whether the the fractional derivatives have a physically reasonable interpretation or not. In addition, the fractional derivatives are very sensitive to the initial and final conditions, which makes the handling of the dynamic equation difficult. Onsager and Machlup ${ }^{25}$ derived the action functional which gives the probability of the fluctuation around the most probable pathway. Although their action functional can be utilized to obtain the information on the dynamics of dissipative systems, it is not the generalization of the action integral in the analytic mechanics. The Onsager-Machlup action should be interpreted as the statistical weight factor for the Brownian motion. Anyway, these methods are formal and mathematical, rather than intuitive and physical. As Bauer ${ }^{26)}$ showed, it is impossible to construct a variational principle for dissipative systems, as a direct and natural generalization of the Hamilton's principle (1).

Some methods and equations are still useful even if they are not in a variational form. Recently Doi ${ }^{27,28)}$ demonstrated that some dynamic equations for soft matters can be obtained by the Onsager's method. It would be informative to critically and carefully examine the Onsager's method from the view point of modern statistical physics. In the nonequilibrium statistical physics, even without a variational principle, we can systematically describe the dynamics near equilibrium. ${ }^{29)}$ If the target system is not far from equilibrium, the dynamics of the system can be described by the equilibrium free energy (the thermodynamic potential) and the mobility tensor. Such a system is often called the linear nonequilibrium system, and we can apply the linear response theory to calculate the mobility. ${ }^{30)}$ The relation between this standard method and the Onsager's method is not fully clear. In addition, as we stated, whether a method is covariant or not is not trivial. We should carefully examine the covariant property of a method. 
In this work, we apply the stochastic energetics ${ }^{2)}$ to the dynamic equation in linear nonequilibrium systems, and derive a balance equation for the free energy change rate and the heat flow from the bath to the system. We consider a general mesoscopic system of which time evolution is given as the Langevin equation. Especially, we show that the dissipative part of the heat flow from the bath to the system plays an important role, and it can be related to Rayleigh's dissipation function. We discuss how we should interpret the Onsager's method, and then we show that we can construct the dynamic equation in an alternative way. The dissipative heat flow is in a covariant form and can be used to derive the mobility tensor. We show that we can systematically construct an equation in a way which is similar to but different from the Onsager's method. We also show a simple coarse-graining procedure for linear nonequilibrium systems, based on the dissipative heat flow. Finally, we apply our general method to some simple systems, as examples. The mesoscopic models such as the dumbbell model and the interacting Brownian particles can be consistently handled by our method.

\section{MODEL AND RESULTS}

\subsection{Dynamics Model}

We start from a general dynamics model for a linear nonequilibrium system, where we do not have reversible currents nor memory kernels. The time evolution of the system can be described reasonably by the Fokker-Planck equation: ${ }^{31)}$

$$
\begin{aligned}
& \frac{\partial P(\boldsymbol{x}, t)}{\partial t} \\
& =\frac{\partial}{\partial x_{i}}\left[L_{i j}(\boldsymbol{x})\left[\frac{\partial \mathcal{F}(\boldsymbol{x})}{\partial x_{j}} P(\boldsymbol{x}, t)+k_{B} T \frac{\partial P(\boldsymbol{x}, t)}{\partial x_{j}}\right]\right],
\end{aligned}
$$

where $\boldsymbol{x}=\left\{x_{i}\right\}$ with $x_{i}$ being the $i$-th slow variable $(i=$ $1,2, \ldots, n), L_{i j}$ is the mobility tensor (the Onsager coefficient), $\mathcal{F}(\boldsymbol{x})$ is the free energy of the system, $k_{B}$ is the Boltzmann coefficient, and $T$ is the temperature. The mobility tensor can depend on $\boldsymbol{x}$, but we require that it does not explicitly depend on time $t$. (If the mobility explicitly depends on time, the analyses become quite complicated. ${ }^{32-34)}$ ) We assume that the Einstein convention is applied to repeated indices (the summations are taken for $i$ and $j$ in eq (4)). The free energy can calculated from the partial partition function for the microscopic degrees of freedom:

$$
e^{-\mathcal{F}(\boldsymbol{x}) / k_{B} T}=\int d \boldsymbol{\Gamma} \delta(\boldsymbol{x}-\boldsymbol{X}(\boldsymbol{\Gamma})) e^{-\mathcal{H}(\boldsymbol{\Gamma}) / k_{B} T},
$$

where $\Gamma$ represents the microscopic degrees of freedom, $\mathcal{H}(\boldsymbol{\Gamma})$ is the Hamiltonian, and $\boldsymbol{X}(\boldsymbol{\Gamma})$ is an expression of the slow variable as a function of the microscopic variable. From the Onsager's reciprocal theorem, ${ }^{3,23,24,27)} L_{i j}$ is symmetric, $L_{i j}=L_{j i}$.

We rewrite the Fokker-Planck equation (4) into the Stratonovich type Langevin equation. (The formalism of stochastic energetics requires the Langevin equation to be the Stratonovich type. ${ }^{2)}$ The Ito type Langevin equation should be first rewritten as the Stratonovich type, in prior to the analyses in the followings.) We express the fluctuating slow variable as $\boldsymbol{X}(t)=\left\{X_{i}(t)\right\}(i=1,2, \ldots, n)$. From eq (4), we have the Stratonovich type Langevin equation as

$$
\begin{aligned}
\frac{d X_{i}(t)}{d t}= & -L_{i j}(\boldsymbol{X}) \frac{\partial \mathcal{F}(\boldsymbol{X})}{\partial X_{j}}+k_{B} T B_{i k}(\boldsymbol{X}) \frac{\partial B_{j k}(\boldsymbol{X})}{\partial X_{j}} \\
& +\sqrt{2 k_{B} T} B_{i j}(\boldsymbol{X}) w_{j}^{(\mathrm{S})}(t),
\end{aligned}
$$

where $B_{i j}(\boldsymbol{X})$ is the noise coefficient tensor which satisfies $B_{i k}(\boldsymbol{X}) B_{j k}(\boldsymbol{X})=L_{i j}(\boldsymbol{X})$, and $w_{i}^{(\mathrm{S})}(t)$ is the Gaussian white noise. We express the Stratonovich type stochastic term by the superscript " $(\mathrm{S})$ ". The noise $w_{i}^{(\mathrm{S})}(t)$ should satisfy the fluctuation-dissipation relation:

$$
\left\langle w_{i}^{(\mathrm{S})}(t)\right\rangle=0, \quad\left\langle w_{i}^{(\mathrm{S})}(t) w_{j}^{(\mathrm{S})}\left(t^{\prime}\right)\right\rangle=\delta_{i j} \delta\left(t-t^{\prime}\right) .
$$

For some analyses and numerical calculations, the Ito type Langevin equation is preferred. The Ito type Langevin equation for eq (6) becomes ${ }^{4,29)}$

$$
\begin{aligned}
\frac{d X_{i}(t)}{d t}= & -L_{i j}(\boldsymbol{X}) \frac{\partial \mathcal{F}(\boldsymbol{X})}{\partial X_{j}}+k_{B} T \frac{\partial L_{i j}(\boldsymbol{X})}{\partial X_{j}} \\
& +\sqrt{2 k_{B} T} B_{i j}(\boldsymbol{X}) w_{j}^{(\mathrm{I})}(t)
\end{aligned}
$$

where $w_{i}^{(\mathrm{I})}(t)$ is the Gaussian white noise, and we use the superscript "(I)" to express the Ito type stochastic term. The noise $w_{i}^{(\mathrm{I})}(t)$ satisfies the same fluctuation-dissipation relation as one for the Stratonovich type Langevin equation: $\left\langle w_{i}^{(\mathrm{I})}(t)\right\rangle=$ 0 and $\left\langle w_{i}^{(\mathrm{I})}(t) w_{j}^{(\mathrm{I})}\left(t^{\prime}\right)\right\rangle=\delta_{i j} \delta\left(t-t^{\prime}\right)$.

The fluctuation can be negligibly small for macroscopic systems. For such a case, we may simply drop the noise term and the associated drift term in the Langevin equation. The resulting dynamic equation is deterministic and common for both the Stratonovich and Ito type equations:

$$
\frac{d X_{i}(t)}{d t}=-L_{i j}(\boldsymbol{X}) \frac{\partial \mathcal{F}(\boldsymbol{X})}{\partial X_{j}}
$$

In this work, we are rather interested in mesoscopic systems 
where we observe the thermal fluctuations. In what follows, therefore, we mainly focus on the mesoscopic systems and do not drop the noise term.

\subsection{Energy Balance}

Following Sekimoto, ${ }^{2,35)}$ we consider the energetics of this system. The energy balance equation for the Langevin equation (6) can be calculated rather straightforwardly. The change of the free energy, $d \mathcal{F}$, is calculated as

$$
\begin{aligned}
d \mathcal{F}= & \frac{\partial \mathcal{F}(\boldsymbol{X})}{\partial X_{i}} d X_{i} \\
= & -\left[\frac{d X_{i}(t)}{d t}-k_{B} T B_{i k}(\boldsymbol{X}) \frac{\partial B_{l k}(\boldsymbol{X})}{\partial X_{l}}\right. \\
& \left.-\sqrt{2 k_{B} T} B_{i k}(\boldsymbol{X}) w_{k}^{(\mathrm{S})}(t)\right] L_{i j}^{-1}(\boldsymbol{X}) d X_{j} .
\end{aligned}
$$

The right hand side can be interpreted as the microscopic heat, $d^{\prime} Q$, which flows from the heat bath (or the environment) to the system. (We use symbol $d^{\prime} Q$ instead of $d Q$ since this quantity is not an exact differential form.) Thus we have

$$
d \mathcal{F}=d^{\prime} Q=d^{\prime} Q_{d}+d^{\prime} Q_{f}
$$

with

$$
\begin{aligned}
& d^{\prime} Q_{d}=-\frac{d X_{i}}{d t} L_{i j}^{-1}(\boldsymbol{X}) d X_{j}, \\
& d^{\prime} Q_{f}=\left[k_{B} T \frac{\partial B_{l k}(\boldsymbol{X})}{\partial X_{l}}+\sqrt{2 k_{B} T} w_{k}^{(\mathrm{S})}(t)\right] B_{k j}^{-1}(\boldsymbol{X}) d X_{j} .
\end{aligned}
$$

We have decomposed the microscopic heat $d^{\prime} Q$ into two components, $d^{\prime} Q_{d}$ and $d^{\prime} Q_{f} . d^{\prime} Q_{d}$ and $d^{\prime} Q_{f}$ represent the dissipative and fluctuating parts of the microscopic heat, respectively. The dissipative heat $d^{\prime} Q_{d}$ is common for the mesoscopic and macroscopic systems, but the fluctuating heat $d^{\prime} Q_{f}$ is absence in the macroscopic system $\left(d^{\prime} Q_{f}=0\right)$. This is because the fluctuating heat originates from the noise term in the Langevin equation.

In some cases, the change rate of the free energy (the time derivative of the free energy) would be convenient than the free energy change itself. Thus we consider the rate of change of the free energy, $d \mathcal{F} / d t$. Then we have

$$
\begin{aligned}
& \frac{d \mathcal{F}(\boldsymbol{X}(t))}{d t}=\frac{d^{\prime} \boldsymbol{Q}(\boldsymbol{X}(t), d \boldsymbol{X}(t) / d t, \boldsymbol{w}(t))}{d t} \\
& =\dot{\boldsymbol{Q}}_{d}\left(\boldsymbol{X}(t), \frac{d \boldsymbol{X}(t)}{d t}\right)+\dot{\boldsymbol{Q}}_{f}\left(\boldsymbol{X}(t), \frac{d \boldsymbol{X}(t)}{d t}, \boldsymbol{w}(t)\right),
\end{aligned}
$$

with

$$
\begin{aligned}
& \dot{Q}_{d}(\boldsymbol{X}, \dot{\boldsymbol{X}})=-\dot{X}_{i} L_{i j}^{-1}(\boldsymbol{X}) \dot{X}_{j}, \\
& \dot{Q}_{f}(\boldsymbol{X}, \dot{\boldsymbol{X}}, \boldsymbol{w}) \\
& =\left[k_{B} T \frac{\partial B_{i k}(\boldsymbol{X})}{\partial X_{i}}+\sqrt{2 k_{B} T} w_{k}^{(\mathrm{S})}\right] B_{i j}^{-1}(\boldsymbol{X}) \dot{X}_{j} .
\end{aligned}
$$

Here, $\dot{Q}_{d}(\boldsymbol{X}, \dot{\boldsymbol{X}})$ and $\dot{\boldsymbol{Q}}_{f}(\boldsymbol{X}, \dot{\boldsymbol{X}}, \boldsymbol{w})$ correspond to the dissipative and fluctuating heat flows from the bath to the system, respectively, for given $\boldsymbol{X}, \dot{\boldsymbol{X}}$, and $\boldsymbol{w}$. The positive definiteness of the mobility tensor $L_{i j}$ ensures the negativity of the dissipative heat flow, $\dot{Q}_{d}(\boldsymbol{X}, \dot{\boldsymbol{X}}) \leq 0$, for any $\boldsymbol{X}$ and $\dot{\boldsymbol{X}}$. The energy balance equation (11) can be rewritten as

$$
\begin{aligned}
& \frac{\partial \mathcal{F}(\boldsymbol{X}(t))}{\partial X_{i}(t)} \dot{X}_{i}(t)=\dot{Q}(\boldsymbol{X}(t), \dot{\boldsymbol{X}}(t)) \\
& =\dot{Q}_{d}(\boldsymbol{X}(t), \dot{\boldsymbol{X}}(t))+\dot{Q}_{f}(\boldsymbol{X}(t), \dot{\boldsymbol{X}}(t)),
\end{aligned}
$$

where $\dot{Q}(\boldsymbol{X}, \dot{\boldsymbol{X}})$ represents the total heat flow and $\dot{\boldsymbol{X}}(t)=$ $d \boldsymbol{X}(t) / d t$.

In some literature, Rayleigh's dissipation function is employed to express the dissipation rate of the energy. ${ }^{9}, 11,12,27$ ) The Reyleigh dissipation function is defined as a function of $\boldsymbol{X}$ and $\dot{\boldsymbol{X}}$ as

$$
\Psi(\boldsymbol{X}, \dot{\boldsymbol{X}}) \equiv \frac{1}{2} \dot{X}_{i} L_{i j}^{-1}(\boldsymbol{X}) \dot{X}_{j}
$$

Comparing eqs (15) and (18), we find

$$
\dot{Q}_{d}(\boldsymbol{X}, \dot{\boldsymbol{X}})=-2 \Psi(\boldsymbol{X}, \dot{\boldsymbol{X}})
$$

This means that, the Rayleigh dissipation function represents the half of the heat flow from the system to the bath, in absence of the fluctuating contribution. This is consistent with the standard interpretation of the Rayleigh dissipation function. ${ }^{9}$ The numerical factor 2 in eq (19) comes from the fact that Rayleigh integrated the heat change with respect to $\dot{X}_{i}$, in the following way:

$$
\Psi(\boldsymbol{X}, \dot{\boldsymbol{X}})=\int_{0}^{\dot{X}_{i}} \dot{X}_{i} L_{i j}(\boldsymbol{X}) d \dot{X}_{j}
$$

so that its partial derivative gives the dissipative force, $-\partial \Psi(\boldsymbol{X}, \dot{\boldsymbol{X}}) / \partial \dot{X}_{i}=-L_{i j} \dot{X}_{j} \cdot{ }^{11,12)} \mathrm{Eq}(20)$ is in analogy to the integration of the thermodynamic force $-\partial \mathcal{F}(X) / \partial X_{i}$ with respect to the variable $X_{i}$, to obtain the thermodynamic potential $\mathcal{F}(\boldsymbol{X})$. However, from eq (12) we know that the microscopic dissipative heat change $d^{\prime} Q_{d}$ is defined for the small change of 
$\boldsymbol{X}$, not for the small change of $\dot{\boldsymbol{X}}$. In addition, the dissipative heat change is not an exact differential form and it seems not to be reasonable to consider an integral quantity like eq (20). Thus the Rayleigh dissipation function should be interpreted as a result of a mathematical trick, rather than a physically meaningful quantity.

The Rayleigh dissipation function is utilized to describe the dynamic equation (without fluctuations) and is sometimes called the "variational" form or the Onsager's "variational" principle: ${ }^{27)}$

$$
\left.\frac{\partial}{\partial \dot{X}_{i}}\left[\frac{\partial \mathcal{F}(\boldsymbol{X})}{\partial X_{j}} \dot{X}_{j}+\Psi(\boldsymbol{X}, \dot{\boldsymbol{X}})\right]\right|_{\dot{\boldsymbol{X}}=d \boldsymbol{X} / d t}=0
$$

It should be noticed that $\dot{X}$ is assumed to be independent of $\boldsymbol{X}$, when we calculate the partial differential with respect to $\dot{X}$ in eq (21). Such an equation cannot be expressed in a variational form as the Hamilton's principle (1). Thus, although eq (21) is refereed as the "variational" form in some literature, in reality it is the minimization condition with respect to $\dot{X}$ rather than the variational principle for $\boldsymbol{X}(t)$. As Bauer ${ }^{26)}$ showed, the simple variational principle, which is a direct generalization of the Hamilton's principle in the analytical mechanics, does not exist for such a system, unless we introduce some mathematical tricks which cannot be physically justified. Since the Rayleigh dissipation function has no reasonable physical meaning, eq (21) should also be interpreted as just a formal mathematical expression.

We consider an alternative equation from the view point of the stochastic energetics. From eq (17), what we should use is the following equation, instead:

$$
\left.\left[\frac{\partial \mathcal{F}(\boldsymbol{X})}{\partial X_{j}} \dot{X}_{j}-\dot{Q}(\boldsymbol{X}, \dot{\boldsymbol{X}}, \boldsymbol{w})\right]\right|_{\dot{\boldsymbol{X}}=d \boldsymbol{X} / d t}=0
$$

$\mathrm{Eq}(22)$ is not a variational form, nor a minimization condition with respect to $\dot{\boldsymbol{X}}$. As clear from its derivation, it is nothing but the energy balance equation. If we ignore the fluctuating heat flow $\left(\dot{Q}_{f}=0\right.$ and thus $\left.\dot{Q}=\dot{Q}_{d}\right)$, eq (22) can be rewritten as

$$
\left.\left[\frac{\partial \mathcal{F}(\boldsymbol{X})}{\partial X_{j}}+\dot{X}_{i} L_{i j}^{-1}\right] \dot{X}_{j}\right|_{\dot{\boldsymbol{X}}=d \boldsymbol{X} / d t}=0
$$

Eq (23) accidentally gives the same equation as eq (21). This partly supports the use of the Rayleigh dissipation function and the minimization condition with respect to $\dot{\boldsymbol{X}}$.

Here we seek a physically reasonable method to construct the dynamic equation. A key point is that the dissipative heat flow (15) is in the bilinear form in $\dot{\boldsymbol{X}}$. Thus from the dissipative heat flow, we can calculate the (inverse) mobility tensor. The mobility tensor can be obtained if we express the dissipative heat flow into the bilinear form in $\dot{X}$, as eq (15). Or, if the explicit form of the dissipative heat flow is given, the following form may be simple and convenient:

$$
L_{i j}^{-1}(\boldsymbol{X})=-\frac{1}{2} \frac{\partial^{2} \dot{Q}_{d}(\boldsymbol{X}, \dot{\boldsymbol{X}})}{\partial \dot{X}_{i} \partial \dot{X}_{j}}
$$

One may consider that the mobility tensor $L_{i j}$ calculated by eq (24) can depend on $\dot{\boldsymbol{X}}$, if the heat flow depends on $\dot{\boldsymbol{X}}$ in a complex way. If the mobility tensor depends on $\dot{\boldsymbol{X}}$, it is beyond the linear nonequilibrium and we cannot construct the Langevin equation which is justified only for linear nonequilibrium systems. Thus the mobility tensor should be independent of $\dot{\boldsymbol{X}}$. Once the mobility tensor is obtained, we can construct the Langevin equation (6) from the mobility tensor $L_{i j}(X)$ and the free energy $\mathcal{F}(\boldsymbol{X})$. In other words, we can construct the dynamic equation from two scalar functions, $\dot{Q}_{d}(\boldsymbol{X}, \dot{\boldsymbol{X}})$ and $\mathcal{F}(\boldsymbol{X})$.

\subsection{Variable Transform and Covariant Property}

Although the Rayleigh dissipation function is not physically sound, it is a scalar quantity and thus eq (21) seems to be essentially independent of the specific choice of the variable $\boldsymbol{X}$ (covariant). We check whether the relations shown above hold under the variable transform from $\boldsymbol{X}$ to $\boldsymbol{X}^{\prime}$ or not. The basic laws of physics should be independent of the specific choice of the variable and thus covariant (which is especially clear in the theory of relativity ${ }^{36)}$ ).

The new slow variable $\boldsymbol{X}^{\prime}$ can be interpreted as a function of $\boldsymbol{X}$, as $\boldsymbol{X}^{\prime}(\boldsymbol{X})$. Conversely, the original slow variable $\boldsymbol{X}$ can be interpreted as $\boldsymbol{X}\left(\boldsymbol{X}^{\prime}\right)$, in a similar way. We introduce the Jacobian matrix as $J_{i j} \equiv \partial X_{i}^{\prime} / \partial X_{j}$, and its inverse becomes $J_{i j}^{-1}=\partial X_{i} / \partial X_{j}^{\prime}$. Then, from the Langevin equation (6), we have

$$
\begin{aligned}
\frac{d X_{i}^{\prime}(t)}{d t}= & J_{i k}\left[-L_{k j}(\boldsymbol{X}) \frac{\partial \mathcal{F}(\boldsymbol{X})}{\partial X_{i}}+k_{B} T B_{k l}(\boldsymbol{X}) \frac{\partial B_{j l}(\boldsymbol{X})}{\partial X_{j}}\right. \\
& \left.+\sqrt{2 k_{B} T} B_{k j}(\boldsymbol{X}) w_{j}^{(\mathrm{S})}(t)\right] \\
= & -L_{j l}^{\prime} \frac{\partial \mathcal{F}^{\prime}}{\partial X_{j}^{\prime}}+k_{B} T B_{i k}^{\prime} \frac{\partial B_{j k}^{\prime}}{\partial X_{j}^{\prime}}+\sqrt{2 k_{B} T} B_{j l}^{\prime} w_{l}^{(\mathrm{S})}(t)
\end{aligned}
$$

where

$$
\mathcal{F}^{\prime}\left(\boldsymbol{X}^{\prime}\right)=\mathcal{F}\left(\boldsymbol{X}\left(\boldsymbol{X}^{\prime}\right)\right)+k_{B} T \ln (\operatorname{det} \boldsymbol{J})
$$




$$
L_{i j}^{\prime}\left(X^{\prime}\right)=J_{i k} L_{k l} J_{l j}, \quad B_{i j}^{\prime}\left(X^{\prime}\right)=J_{i k} B_{k j} .
$$

Thus we find that the Langevin equation (6) can be transformed into the same form by the variable transform. However, it should be noticed that the free energy $\mathcal{F}(\boldsymbol{X})$ in eq (6) is generally different from $\mathcal{F}^{\prime}\left(\boldsymbol{X}^{\prime}\right)$ in eq (25). That is, the free energy is not in a covariant form. Although this may not look intuitive, it is consistent with the equilibrium statistical mechanics. The free energy for $\boldsymbol{X}^{\prime}$ should be defined as

$$
e^{-\mathcal{F}^{\prime}\left(\boldsymbol{x}^{\prime}\right) / k_{B} T} \equiv \int d \boldsymbol{x} \delta\left(\boldsymbol{x}^{\prime}-\boldsymbol{X}^{\prime}(\boldsymbol{x})\right) e^{-\mathcal{F}(\boldsymbol{x}) / k_{B} T}
$$

As Nakamura ${ }^{37)}$ explicitly stated, the free energy $\mathcal{F}^{\prime}$ generally depends on the Jacobian matrix for the variable transform. Eq (28) can be modified as

$$
e^{-\mathcal{F}^{\prime}\left(\boldsymbol{x}^{\prime}\right) / k_{B} T}=\frac{1}{\operatorname{det} \boldsymbol{J}} e^{-\mathcal{F}\left(\boldsymbol{X}\left(\boldsymbol{x}^{\prime}\right)\right) / k_{B} T},
$$

and this gives eq (26). Eqs (26) and (29) mean that the free energy is not in a covariant form. Thus, when we transform the slow variable, we should carefully calculate the free energy for the new slow variable.

The energy balance equation is

$$
\begin{aligned}
& \frac{d \mathcal{F}^{\prime}\left(X^{\prime}(t)\right)}{d t} \\
& =\dot{Q}_{d}^{\prime}\left(\boldsymbol{X}^{\prime}(t), \frac{d \boldsymbol{X}^{\prime}(t)}{d t}\right)+\dot{Q}_{f}^{\prime}\left(\boldsymbol{X}^{\prime}(t), \frac{d \boldsymbol{X}^{\prime}(t)}{d t}, \boldsymbol{w}(t)\right),
\end{aligned}
$$

with

$$
\begin{aligned}
& \dot{Q}_{d}^{\prime}\left(\boldsymbol{X}^{\prime}, \dot{X}^{\prime}\right)=-\dot{X}_{i}^{\prime} L_{i j}^{\prime-1}\left(\boldsymbol{X}^{\prime}\right) \dot{X}_{j}^{\prime}, \\
& \dot{Q}_{f}^{\prime}\left(\boldsymbol{X}^{\prime}, \dot{\boldsymbol{X}}^{\prime}, \boldsymbol{w}\right) \\
& =\left[k_{B} T \frac{\partial B_{i k}^{\prime}\left(\boldsymbol{X}^{\prime}\right)}{\partial X_{k}^{\prime}}+\sqrt{2 k_{B} T} w_{i}^{(\mathrm{S})}\right] B_{i j}^{\prime-1}(\boldsymbol{X}) \dot{X}_{j}^{\prime} .
\end{aligned}
$$

From eq (26), the left hand side of eq (30) is not equal to that of eq (14) $\left(d \mathcal{F} / d t \neq d \mathcal{F}^{\prime} / d t\right)$, although the energy balance equation itself holds. This is because the form of the free energy (strictly speaking, the entropy term in the free energy) depends on the choice of the variable. This can be understood that some terms in the right hand side in eq (14) moved to the left hand side in eq (30), under the variable transform. However, the dissipative heat flow is unchanged under this transform:

$$
\dot{Q}_{d}^{\prime}\left(X^{\prime}, \dot{X}^{\prime}\right)=\dot{Q}_{d}(X, \dot{X})
$$

The dissipative heat flow $\dot{Q}_{d}$ is therefore independent of the specific choice of the variable and thus is in a covariant form. Therefore, the mobility tensor for $\boldsymbol{X}^{\prime}$ can be directly obtained from the dissipative heat flow for the original variable, in the same form as eq (24):

$$
L_{i j}^{\prime-1}\left(\boldsymbol{X}^{\prime}\right)=-\frac{1}{2} \frac{\partial^{2} \dot{Q}_{d}\left(\boldsymbol{X}^{\prime}, \dot{X}^{\prime}\right)}{\partial \dot{X}_{i}^{\prime} \partial \dot{X}_{j}^{\prime}}
$$

Thus we conclude that, without explicitly calculating the variable transform for the Langevin equation, we can construct the Langevin equation for a given form of the slow variable, only from eqs (26) and (34). A procedure to derive the Langevin equation for a given variable $\boldsymbol{X}$ can be summarized as follows.

1. Calculate the free energy $\mathcal{F}(\boldsymbol{X})$. This is independent of the details of dynamics, and can be calculated from the partial partition function as eq (5). (However, the free energy is not covariant and it should be calculated for this specific variable $\boldsymbol{X}$, not for other variables.)

2. Calculate the dissipative heat flow $\dot{Q}_{d}$. This quantity is covariant (independent of the choice of the variable), and thus any variable transforms can be used to calculate it.

3. Calculate the mobility tensor $L_{i j}(\boldsymbol{X})$ from $\dot{Q}_{d}$ via eq (24), and then construct the Langevin equation from $\mathcal{F}(\boldsymbol{X})$ and $L_{i j}(X)$. Both the Stratonovich and the Ito types (eqs (6) and (8)) can be employed.

This is a general method to construct the mesoscopic dynamic equation, and is one of the main results in this work. Following this method, we can utilize the covariant property and construct the dynamic equation systematically.

Here, it would be informative to mention about so-called the Rayleighian. Eq (21) is sometimes rewritten by introducing the Rayleighian $\mathcal{R}:{ }^{27)}$

$$
\begin{aligned}
& \mathcal{R}(\boldsymbol{X}, \dot{\boldsymbol{X}}) \equiv \frac{\partial \mathcal{F}(\boldsymbol{X})}{\partial X_{j}} \dot{X}_{j}+\Psi(\boldsymbol{X}, \dot{\boldsymbol{X}}) \\
&=\frac{\partial \mathcal{F}(\boldsymbol{X})}{\partial X_{j}} \dot{X}_{j}+\frac{1}{2} \dot{X}_{i} L_{i j}^{-1} \dot{X}_{j} \\
&\left.\frac{\partial \mathcal{R}(\boldsymbol{X}, \dot{\boldsymbol{X}})}{\partial \dot{\boldsymbol{X}}}\right|_{\dot{\boldsymbol{X}}=d \boldsymbol{X} / d t}=0
\end{aligned}
$$

As we showed, the dissipative heat flow $\dot{Q}_{d}$ is in a covariant form (eq (33)), and from eq (19), the Rayleigh dissipation function $\Psi$ is also in a covariant form. However, the free energy $\mathcal{F}$ is not in a covariant form, as clearly shown in eq (26). Therefore, the Rayleighian varies under the variable transform 
and $\mathcal{R}^{\prime}\left(\boldsymbol{X}^{\prime}, \dot{\boldsymbol{X}}^{\prime}\right) \neq \mathcal{R}(\boldsymbol{X}, \dot{\boldsymbol{X}})$, in general. As a result, the minimization condition (36) is not in a covariant form, neither. This means that, if we calculate the Rayleighian for a specific variable $\boldsymbol{X}$ as $\mathcal{R}(\boldsymbol{X}, \dot{\boldsymbol{X}})$ but minimize it with respect to other variable $\boldsymbol{X}^{\prime}$ as $\partial \mathcal{R}(\boldsymbol{X}, \dot{\boldsymbol{X}}) /\left.\partial \dot{\boldsymbol{X}}^{\prime}\right|_{\dot{\boldsymbol{X}}^{\prime}=d \boldsymbol{X}^{\prime} / d t}=0$, the resulting dynamic equation for $\boldsymbol{X}^{\prime}$ is not correct (unless the Jacobian matrix is independent of $\boldsymbol{X}^{\prime}$ ). The Onsager's "variational" method which utilizes the Rayleighian is, therefore, not variational nor covariant. (Strictly speaking, the Rayleigh's original method which utilizes the microscopic Lagrangian and the dissipation function is covariant, since the Lagraigian is covariant. But of course, it is not variational.)

\subsection{Coarse-Graining}

The dissipative heat flow may be also utilized for the coarse-graining. To obtain the coarse-grained dynamic equation, we statistically eliminate relatively fast variables. Because the choice of the slow variable is rather arbitrary, we set $\boldsymbol{X}=\{\boldsymbol{Y}, \boldsymbol{\Theta}\}$, where $\boldsymbol{Y}=\left\{Y_{i}\right\}(i=1,2, \ldots, m)$ is the set of slow variables for the coarse-grained system, and $\boldsymbol{\Theta}=\left\{\Theta_{\alpha}\right\}$ $(\alpha=m+1,2, \ldots, n)$ is the set of the relatively fast variables to be eliminated. In this subsection, we use subscripts $i, j, k, \ldots$ for the coarse-grained slow variables and subscripts $\alpha, \beta, \gamma, \ldots$ for the relatively fast variables.

The free energy for the coarse-grained system $\overline{\mathcal{F}}(\boldsymbol{Y})$ is

$$
e^{-\overline{\mathcal{F}}(\mathbf{Y}) / k_{B} T}=\int d \boldsymbol{\Theta} e^{-\mathcal{F}(\mathbf{Y}, \boldsymbol{\Theta}) / k_{B} T} .
$$

The dissipative heat flow of the system can be expressed as

$$
\begin{aligned}
\dot{Q}_{d} & =-\left[\begin{array}{ll}
\dot{Y}_{i} & \dot{\Theta}_{\alpha}
\end{array}\right]\left[\begin{array}{cc}
M_{i j} & M_{i \beta} \\
M_{\alpha j} & M_{\alpha \beta}
\end{array}\right]\left[\begin{array}{c}
\dot{Y}_{j} \\
\dot{\Theta}_{\beta}
\end{array}\right] \\
& =-\dot{Y}_{i} M_{i j} \dot{Y}_{j}-2 \dot{Y}_{i} M_{i \alpha} \dot{\Theta}_{\alpha}-\dot{\Theta}_{\alpha} M_{\alpha \beta} \dot{\Theta}_{\beta},
\end{aligned}
$$

where we have defined the components of the inverse mobility tensor via

$$
\left[\begin{array}{cc}
M_{i k} & M_{i \gamma} \\
M_{\alpha k} & M_{\alpha \gamma}
\end{array}\right]\left[\begin{array}{cc}
L_{k j} & L_{k \beta} \\
L_{\gamma j} & L_{\gamma \beta}
\end{array}\right]=\left[\begin{array}{cc}
\delta_{i j} & 0 \\
0 & \delta_{\alpha \beta}
\end{array}\right] .
$$

The dissipative heat flow is a function of $\boldsymbol{Y}, \boldsymbol{\Theta}, \dot{\boldsymbol{Y}}$, and $\dot{\boldsymbol{\Theta}}$. We want to eliminate the relatively fast variables $\boldsymbol{\Theta}$ and $\dot{\boldsymbol{\Theta}}$, to obtain the effective dissipative heat flow for the coarse-grained slow variables. For $\boldsymbol{\Theta}$, we can simply take the statistical average over the local equilibrium distribution:

$$
P_{\text {leq }}(\Theta \mid \boldsymbol{Y})=e^{\overline{\mathcal{F}}(\mathbf{Y}) / k_{B} T-\mathcal{F}(\boldsymbol{Y}, \boldsymbol{\Theta}) / k_{B} T}
$$

The equilibrium distribution of $\dot{\boldsymbol{\Theta}}$ is generally not that simple. However, because we employed the local equilibrium distribution for $\boldsymbol{\Theta}$, it would be natural to expect that $\dot{\boldsymbol{\Theta}}$ is characterized by a simple fluctuation around the local equilibrium. It may depend on the slow degrees of freedom, $\boldsymbol{Y}$ and $\dot{\boldsymbol{Y}}$. We can rewrite the dissipative heat flow as follows:

$$
\begin{aligned}
\dot{Q}_{d}= & -\dot{Y}_{i}\left(M_{i j}-M_{i \alpha} M_{\alpha \beta}^{-1} M_{\beta j}\right) \dot{Y}_{j} \\
& -\left(\dot{\Theta}_{\alpha}+M_{\alpha \gamma}^{-1} M_{\gamma i} \dot{Y}_{i}\right) M_{\alpha \beta}\left(\dot{\Theta}_{\beta}+M_{\beta \delta}^{-1} M_{\delta j} \dot{Y}_{j}\right) .
\end{aligned}
$$

Here, we may interpret the second term in the right hand side of eq (41) as the dissipative heat flow due to the relatively fast degrees of freedom. Following this interpretation, we assume the following phenomenological form for the local equilibrium distribution of $\dot{\boldsymbol{\Theta}}$ :

$$
\begin{aligned}
& P_{\text {leq }}(\dot{\boldsymbol{\Theta}} \mid \dot{\boldsymbol{Y}}, \boldsymbol{Y}, \boldsymbol{\Theta}) \\
& =\frac{1}{\sqrt{2 \pi \operatorname{det} \boldsymbol{C}}} e^{-\left(\dot{\Theta}_{\alpha}+M_{\alpha \gamma}^{-1} M_{\gamma i} \dot{Y}_{i}\right) G_{\alpha \beta}^{-1}\left(\dot{\Theta}_{\beta}+M_{\beta \delta}^{-1} M_{\delta j} \dot{Y}_{j}\right) / 2},
\end{aligned}
$$

where $G_{\alpha \beta}(\boldsymbol{Y}, \boldsymbol{\Theta})$ is the covariance matrix, and can depend both on $\boldsymbol{Y}$ and $\boldsymbol{\Theta}$.

Then we have the following approximate form for the coarse-grained dissipative heat flow:

$$
\begin{aligned}
\dot{\bar{Q}}_{d}(\boldsymbol{Y}, \dot{\boldsymbol{Y}}) \approx & \int d \Theta d \dot{\Theta} P_{\mathrm{leq}}(\boldsymbol{\Theta} \mid \boldsymbol{Y}) P_{\mathrm{leq}}(\dot{\boldsymbol{\Theta}} \mid \dot{\boldsymbol{Y}}, \boldsymbol{Y}, \boldsymbol{\Theta}) \dot{\mathbf{Q}}_{d} \\
= & \int d \Theta P_{\mathrm{leq}}(\boldsymbol{\Theta} \mid \boldsymbol{Y})\left[-\dot{Y}_{i}\left(M_{i j}\right.\right. \\
& \left.\left.-M_{i \alpha} M_{\alpha \beta}^{-1} M_{j \beta}\right) \dot{Y}_{j}-G_{\alpha \beta} M_{\alpha \beta}\right] \\
= & -\dot{Y}_{i} \bar{L}_{i j}^{-1} \dot{Y}_{j}+\dot{\bar{Q}}_{d}^{(\mathrm{leq})}(\boldsymbol{Y}),
\end{aligned}
$$

with

$$
\begin{aligned}
& \bar{L}_{i j}^{-1}=\int d \Theta P_{\text {leq }}(\Theta \mid \boldsymbol{Y})\left(M_{i j}-M_{i \alpha} M_{\alpha \beta}^{-1} M_{\beta j}\right), \\
& \dot{\bar{Q}}_{d}^{\text {(leq })}(\boldsymbol{Y})=-\int d \boldsymbol{\Theta} P_{\text {leq }}(\boldsymbol{\Theta} \mid \boldsymbol{Y}) G_{\alpha \beta} M_{\alpha \beta} .
\end{aligned}
$$

Here, $\dot{\bar{Q}}_{d}^{(\text {leq) }}$ is independent of $\dot{\boldsymbol{Y}}$ and does not contribute to the mobility tensor. In addition, it should be balanced to the statistically averaged fluctuating heat flow, otherwise the energy balance equation is violated. Thus we may simply ignore $\dot{\bar{Q}}_{d}^{\text {(leq) }}$. Further, if we assume that the coarse-grained fluctuating heat flow has the same form as eq (16), we recover the energy balance equation which has the same form as eq (14).

Eq (44) gives the coarse-grained mobility tensor. By calculating the mobility tensor for $\boldsymbol{Y}$ by inverting eq (44), and introducing the noise tensor $\bar{B}_{i j}$ via $\bar{B}_{i k} \bar{B}_{j k}=\bar{L}_{i k}$, finally 
we can construct the approximate Langevin equation for the coarse-grained variable:

$$
\begin{aligned}
\frac{d Y_{i}(t)}{d t}= & -\bar{L}_{i j} \frac{\partial \overline{\mathcal{F}}(\boldsymbol{Y})}{\partial Y_{j}}+k_{B} T \bar{B}_{i k}(\boldsymbol{Y}) \frac{\partial \bar{B}_{j k}(\boldsymbol{Y})}{\partial Y_{j}} \\
& +\sqrt{2 k_{B} T} \bar{B}_{i j}(\boldsymbol{Y}) w_{j}^{(\mathrm{S})} .
\end{aligned}
$$

Of course, the obtained Langevin equation (46) by our method is not exact. In a standard coarse-graining procedure, the elimination of relatively fast variables should be conducted with the projection operator method, and the result becomes the generalized Langevin equation with the memory kernel. ${ }^{38)}$ However, the memory kernel is difficult to handle with, and the memoryless Langevin equation under the Markovian approximation is widely employed as an approximate dynamic equation. (A recent work ${ }^{39)}$ showed that the generalized Langevin equation can be approximated by a Langevin equation with an effective mobility tensor, if the memory effect is weak.) Our derivation of the coarse-grained Langevin equation does not use the projection operator and is simple (although the validity and accuracy of approximations are not fully justified). Due to its simplicity, it will be useful for some applications. This result is also one of the main results in this work. Both the construction of the Langevin equation and the coarse-graining can be performed in a similar way, by utilizing the dissipative heat flow.

\section{EXAMPLES}

In Sec 2., we obtained a general method to construct the Langevin equation for the system, and also a general coarsegraining method. These methods are rather formal and it is not clear whether they can be really applied to systems which are interesting from the rheological viewpoint. In this section, we apply our method to some simple systems, as examples, and study how our methods work.

\subsection{Dumbbell model}

As a simple example, we consider the dynamics of a dumbbell model. ${ }^{5)}$ We consider a dumbbell which consists of two particles connected by a bond potential, and express the particle positions as $\boldsymbol{r}_{1}$ and $\boldsymbol{r}_{2}$, and the bond potential as $U\left(\boldsymbol{r}_{1}-\boldsymbol{r}_{2}\right)$. This bond potential can be interpreted as the free energy of the dumbbell. Also, we express the mobility tensor as $L_{i j}\left(\boldsymbol{r}_{1}, \boldsymbol{r}_{2}\right)(i, j=1,2)$. The mobility tensor can depend on $\boldsymbol{r}_{i}$ in a complex way. For example, if the friction coefficient is position-dependent, the elements of the mobility tensor depend on the positions. The motion of the solvent around the dumbbell will be (approximately) expressed as offdiagonal elements. (Strictly speaking, the mobility tensor is generally not isotropic. In this work, for simplicity, we limit ourselves to an isotropic mobility tensor model.) Then, the Langevin equation becomes

$$
\begin{aligned}
\frac{d \boldsymbol{r}_{i}(t)}{d t}= & -L_{i j} \frac{\partial U}{\partial \boldsymbol{r}_{j}}+k_{B} T B_{i k} \frac{\partial B_{j k}}{\partial \boldsymbol{r}_{j}} \\
& +\sqrt{2 k_{B} T} B_{i j} \boldsymbol{w}_{j}^{(\mathrm{S})}(t),
\end{aligned}
$$

where $B_{i j}\left(\boldsymbol{r}_{1}, \boldsymbol{r}_{2}\right)$ is the noise coefficient tensor which satisfies $B_{i k} B_{j k}=L_{i j}$, and $\boldsymbol{w}_{i}^{(\mathrm{S})}(t)$ is the Gaussian white noise which satisfies

$$
\left\langle\boldsymbol{w}_{i}^{(\mathrm{S})}(t)\right\rangle=0, \quad\left\langle\boldsymbol{w}_{i}^{(\mathrm{S})}(t) \boldsymbol{w}_{j}^{(\mathrm{S})}\left(t^{\prime}\right)\right\rangle=\delta_{i j} \mathbf{1} \delta\left(t-t^{\prime}\right) .
$$

Here, 1 represents the unit tensor.

To study the diffusion and relaxation behavior, it is convenient to use the bond vector $\boldsymbol{q}=\boldsymbol{r}_{1}-\boldsymbol{r}_{2}$ and the center of mass $\boldsymbol{R}=\left(\boldsymbol{r}_{1}+\boldsymbol{r}_{2}\right) / 2$, instead of the particle positions. The Jacobian determinant is unity for this variable transform, and thus the potential (the free energy) is not changed. The bond potential is a function of the bond vector and is independent of the center of mass, $\partial U / \partial \boldsymbol{R}=0$. We can rewrite the dynamic equations in terms of $\boldsymbol{q}$ and $\boldsymbol{R}$. We calculate the mobility tensor from the dissipative heat flow by utilizing eq (24) (or eq (34)). The dissipative heat flow is simply calculated to be

$$
\begin{aligned}
\dot{Q}_{d}= & -\frac{1}{L_{11} L_{12}-L_{12}^{2}}\left[L_{22}(\dot{\boldsymbol{R}}-\dot{\boldsymbol{q}} / 2)^{2}\right. \\
& \left.+L_{11}(\dot{\boldsymbol{R}}+\dot{\boldsymbol{q}} / 2)^{2}-2 L_{12}\left(\dot{\boldsymbol{R}}^{2}-\dot{\boldsymbol{q}}^{2} / 4\right)\right] .
\end{aligned}
$$

Then, the inverse mobility tensor for $[\boldsymbol{q} \boldsymbol{R}]^{\mathrm{T}}$ is calculated as

$$
\begin{aligned}
& \boldsymbol{\Lambda}^{-1}=-\frac{1}{2}\left[\begin{array}{ll}
\partial^{2} / \partial \dot{\boldsymbol{q}} \partial \dot{\boldsymbol{q}} & \partial^{2} / \partial \dot{\boldsymbol{q}} \partial \dot{\boldsymbol{R}} \\
\partial^{2} / \partial \dot{\boldsymbol{R}} \partial \dot{\boldsymbol{q}} & \partial^{2} / \partial \dot{\boldsymbol{R}} \partial \dot{\boldsymbol{R}}
\end{array}\right] \dot{\mathbf{Q}}_{d} \\
& =\frac{1}{L_{11} L_{12}-L_{12}^{2}} \\
& \quad \times\left[\begin{array}{cc}
\left(L_{11}+L_{22}+2 L_{12}\right) / 4 & \left(L_{11}-L_{22}\right) / 2 \\
\left(L_{11}-L_{22}\right) / 2 & L_{11}+L_{22}-2 L_{12}
\end{array}\right] \mathbf{1} .
\end{aligned}
$$

By inverting eq (50), we have the mobility tensor as

$$
\boldsymbol{\Lambda}=\left[\begin{array}{cc}
L_{11}+L_{22}-2 L_{12} & \left(L_{22}-L_{11}\right) / 2 \\
\left(L_{22}-L_{11}\right) / 2 & \left(L_{11}+L_{22}+2 L_{12}\right) / 4
\end{array}\right] \mathbf{1}
$$

We introduce the noise coefficient tensor for the mobility tensor (51) as $\boldsymbol{C} \cdot \boldsymbol{C}^{\mathrm{T}}=\boldsymbol{\Lambda}$. We use the Cholesky decomposition 
of $\boldsymbol{\Lambda}$ as the noise coefficient tensor $\boldsymbol{C}$ :

$$
\begin{aligned}
\boldsymbol{C}= & \frac{1}{\sqrt{L_{22}+L_{11}-2 L_{12}}} \\
& \times\left[\begin{array}{cc}
L_{22}+L_{11}-2 L_{12} & 0 \\
\left(L_{22}-L_{11}\right) / 2 & \sqrt{L_{11} L_{22}-L_{12}^{2}}
\end{array}\right] \mathbf{1} .
\end{aligned}
$$

With the thus obtained mobility tensor, noise coefficient tensor, and the bond potential (the free energy), the Langevin equation for the bond vector and the center of mass can be expressed as follows:

$$
\begin{aligned}
\frac{d}{d t}\left[\begin{array}{l}
\boldsymbol{q}(t) \\
\boldsymbol{R}(t)
\end{array}\right]= & -\boldsymbol{\Lambda} \cdot\left[\begin{array}{c}
\partial U / \partial \boldsymbol{q} \\
0
\end{array}\right]+k_{B} T \boldsymbol{C} \cdot\left[\left[\begin{array}{ll}
\frac{\partial}{\partial \boldsymbol{q}} & \frac{\partial}{\partial \boldsymbol{R}}
\end{array}\right] \cdot \boldsymbol{C}\right]^{\mathrm{T}} \\
& +\sqrt{2 k_{B} T} \boldsymbol{C} \cdot\left[\begin{array}{l}
\boldsymbol{w}_{1}^{(\mathrm{S})}(t) \\
\boldsymbol{w}_{2}^{(\mathrm{S})}(t)
\end{array}\right]
\end{aligned}
$$

We should recall that the Langevin equation (53) can be obtained by directly performing the variable transform for the Langevin equation. Therefore, although our approach simplified the calculation somewhat, eq (53) itself is rather trivial. As a non-trivial operation, next we consider the coarse-graining. We eliminate the degrees of freedom for the bond and derive the effective dynamic equation for the center of mass. Such a coarse-graining will be useful when we study the long-time diffusion behavior. From eq (44), we have the following expression for the effective mobility:

$$
\frac{1}{\bar{\Lambda}(\boldsymbol{R})}=\frac{1}{\mathcal{Z}} \int d \boldsymbol{q} \frac{4}{L_{11}+L_{22}+2 L_{12}} e^{-U(\boldsymbol{q}) / k_{B} T} .
$$

with $\mathcal{Z}=\int d \boldsymbol{q} e^{-U(\boldsymbol{q}) / k_{B} T}$. Also, the effective potential (the free energy) $\bar{U}(\boldsymbol{R})$ is calculated as

$$
e^{-\bar{U}(\boldsymbol{R})}=\int d \boldsymbol{q} e^{-U(\boldsymbol{q}) / k_{B} T}
$$

and this gives $\bar{U}(\boldsymbol{R})=-k_{B} T \ln \mathcal{Z}$, which is constant of $\boldsymbol{R}$. Therefore the effective potential does not contribute to any thermodynamic properties, and thus we simply discard it. The resulting coarse-grained Langevin equation is

$$
\frac{d \boldsymbol{R}(t)}{d t}=k_{B} T \sqrt{\bar{\Lambda}(\boldsymbol{R})} \frac{\partial}{\partial \boldsymbol{R}} \sqrt{\bar{\Lambda}(\boldsymbol{R})}+\sqrt{2 k_{B} T \bar{\Lambda}(\boldsymbol{R})} \boldsymbol{w}^{(\mathrm{S})}(t),
$$

where $\boldsymbol{w}^{(\mathrm{S})}(t)$ is a Gaussian random noise which satisfies $\left\langle\boldsymbol{w}^{(\mathrm{S})}(t)\right\rangle=0$ and $\left\langle\boldsymbol{w}^{(\mathrm{S})}(t) \boldsymbol{w}^{(\mathrm{S})}\left(t^{\prime}\right)\right\rangle=\mathbf{1} \delta\left(t-t^{\prime}\right)$. Eq (56) represents a simple diffusion process with the position-dependent mobility $\bar{\Lambda}(\boldsymbol{R})$. The effect of the eliminated degrees of freedom is taken into account via eq (54).

\subsection{Trapped Particle}

As another simple example, we consider a single particle trapped in a central force potential. We express the particle position measured from the trap center as $\boldsymbol{r}$ (in the three dimensional Cartesian coordinate), and the trap potential as $U(\boldsymbol{r})=U(|\boldsymbol{r}|)$. The trap potential can be interpreted as the free energy. We assume that the mobility can depend on the distance from the trap center but is isotropic, and express it as $L(\boldsymbol{r})=L(|\boldsymbol{r}|)$. Then, the Langevin equation for a particle is

$$
\begin{aligned}
\frac{d \boldsymbol{r}(t)}{d t}= & -L(\boldsymbol{r}) \frac{\partial U(\boldsymbol{r})}{\partial \boldsymbol{r}}+k_{B} T \sqrt{L(\boldsymbol{r})} \frac{\partial}{\partial \boldsymbol{r}} \sqrt{L(\boldsymbol{r})} \\
& +\sqrt{2 k_{B} T L(\boldsymbol{r})} \boldsymbol{w}^{(\mathrm{S})}(t),
\end{aligned}
$$

where $\boldsymbol{w}^{(\mathrm{S})}(t)$ is the Gaussian white noise which satisfies $\left\langle\boldsymbol{w}^{(\mathrm{S})}(t)\right\rangle=0$ and $\left\langle\boldsymbol{w}^{(\mathrm{S})}(t) \boldsymbol{w}^{(\mathrm{S})}\left(t^{\prime}\right)\right\rangle=\mathbf{1} \delta\left(t-t^{\prime}\right)$. The dissipative heat flow is simply calculated as

$$
\dot{Q}_{d}=-\frac{\dot{\boldsymbol{r}}^{2}}{L(\boldsymbol{r})}
$$

We consider the dynamic equation in the spherical coordinate $[r, \phi, \theta](r>0,0 \leq \phi<2 \pi$, and $0 \leq \theta \leq \pi)$, defined as $r=|\boldsymbol{r}|, \phi=\tan ^{-1}\left(r_{2} / r_{3}\right)$, and $\theta=\cos ^{-1}\left(r_{3} / r\right)$. The Jacobian determinant is $1 / r^{2} \sin \theta$, and thus the potential at the spherical coordinate can be expressed as

$$
U^{\prime}(r, \theta)=U(r)-k_{B} T \ln \left(r^{2} \sin \theta\right) .
$$

The dissipative heat flow (58) can be rewritten in terms of $[\dot{r}, \dot{\phi}, \dot{\theta}]$ as

$$
\dot{Q}_{d}=-\frac{1}{L(r)}\left[\dot{r}^{2}+r^{2} \sin ^{2} \theta \dot{\phi}^{2}+r^{2} \dot{\theta}^{2}\right]
$$

and thus the inverse mobility tensor for $[r, \phi, \theta]^{\mathrm{T}}$ becomes

$$
\boldsymbol{L}^{\prime-1}=\frac{1}{L(r)}\left[\begin{array}{ccc}
1 & 0 & 0 \\
0 & r^{2} \sin ^{2} \theta & 0 \\
0 & 0 & r^{2}
\end{array}\right]
$$

By inverting eq (61), we have the mobility tensor and the noise coefficient tensor as

$$
\begin{aligned}
& \boldsymbol{L}^{\prime}=L(r)\left[\begin{array}{ccc}
1 & 0 & 0 \\
0 & 1 / r^{2} \sin ^{2} \theta & 0 \\
0 & 0 & 1 / r^{2}
\end{array}\right], \\
& \boldsymbol{B}^{\prime}=\sqrt{L(r)}\left[\begin{array}{ccc}
1 & 0 & 0 \\
0 & 1 / r \sin \theta & 0 \\
0 & 0 & 1 / r
\end{array}\right] .
\end{aligned}
$$


Therefore, the Langevin equations for $r, \phi$, and $\theta$ become

$$
\begin{aligned}
\frac{d r(t)}{d t}= & -L(r)\left[\frac{d U(r)}{d r}-\frac{2 k_{B} T}{r}\right]+\frac{k_{B} T}{2} \frac{d L(r)}{d r} \\
& +\sqrt{2 k_{B} T L(r)} w_{1}^{(\mathrm{S})}(t), \\
\frac{d \phi(t)}{d t}= & \frac{\sqrt{2 k_{B} T L(r)}}{r \sin \theta} w_{2}^{(\mathrm{S})}(t), \\
\frac{d \theta(t)}{d t}= & -\frac{L(r)}{r^{2}}\left(-\frac{k_{B} T}{\tan \theta}\right)+\frac{\sqrt{2 k_{B} T L(r)}}{r} w_{3}^{(\mathrm{S})}(t) .
\end{aligned}
$$

The potential $U^{\prime}(r, \theta)$ gives an additional force term for $r$ arising from the Jacobian determinant (Neumann's radial force ${ }^{40)}$ ), as clearly found in eq (63). This additional force diverges at $r \rightarrow 0$, and thus it repels the particle from the trap center. We observe a similar additional force term for $\theta$ in eq (65). The derivation of eqs (63)-(64) based on our procedure is straightforward, and free from many complicated and lengthy calculations for derivatives in spherical coordinates.

Next, we consider to derive an approximate dynamic equation for the distance from the trap center, $r$. This can be achieved by the coarse-graining, in which the remaining degrees of freedom, $\phi$ and $\theta$, are eliminated. The effective potential for $r, \bar{U}(r)$, is calculated as

$$
\begin{aligned}
e^{-\bar{U}(r) / k_{B} T}= & \int d \phi d \theta r^{2} \sin \theta e^{-U(r) / k_{B} T} \\
& =4 \pi r^{2} e^{-U(r) / k_{B} T}
\end{aligned}
$$

and we have $\bar{U}(r)=U(r)-k_{B} T \ln r^{2}+$ (const.). The terms in the dissipative heat flow (58) are already decoupled, thus we simply have the effective mobility $\bar{L}(r)$ for $r$ as

$$
\frac{1}{\bar{L}(r)}=\int d \phi d \theta \frac{\sin \theta}{4 \pi} \frac{1}{L(r)}=\frac{1}{L(r)} .
$$

Finally, we have the following Langevin equation as an approximate dynamic equation for $r$ :

$$
\begin{aligned}
\frac{d r(t)}{d t}= & -L(r)\left[\frac{d U(r)}{d r}-\frac{2 k_{B} T}{r}\right]+\frac{k_{B} T}{2} \frac{d L(r)}{d r} \\
& +\sqrt{2 k_{B} T L(r)} w^{(\mathrm{S})}(r),
\end{aligned}
$$

where $w^{(\mathrm{S})}(t)$ is the Gaussian white noise which satisfies $\left\langle w^{(\mathrm{S})}(t)\right\rangle=0$ and $\left\langle w^{(\mathrm{S})}(t) w^{(\mathrm{S})}\left(t^{\prime}\right)\right\rangle=\delta\left(t-t^{\prime}\right)$. Eq (68) has the same form as eq (63). Because the dynamics of $\theta$ and $\phi$ does not affect that of $r$, this result is reasonable.

We consider other approximate dynamic equations, by eliminating the distance $r$. This is another coarse-graining and the effective directional dynamics will be obtained. The effective potential for $\phi$ and $\theta$ is

$$
e^{-\bar{U}(\phi, \theta)}=\sin \theta \int d r r^{2} e^{-U(r) / k_{B} T}
$$

and thus $\bar{U}(\phi, \theta)=-k_{B} T \ln \sin \theta+$ (const.). (The same effective potential was utilized by Doi, ${ }^{27)}$ although he showed the free energy for the distribution which also has the entropic contribution.) The effective mobility tensor $\overline{\boldsymbol{L}}$ for $[\phi, \theta]^{\mathrm{T}}$ is given as

$$
\begin{aligned}
\overline{\boldsymbol{L}}^{-1}= & \frac{1}{\int d r r^{2} e^{-U(r) / k_{B} T}} \int d r r^{2} e^{-U(r) / k_{B} T} \\
& \times \frac{1}{L(r)}\left[\begin{array}{cc}
r^{2} \sin ^{2} \theta & 0 \\
0 & r^{2}
\end{array}\right] .
\end{aligned}
$$

Although we cannot calculate the integrals over $r$ unless the potential $U(r)$ is specified, the $r$-dependent part can be separated and we have the following general form:

$$
\overline{\boldsymbol{L}}=\bar{\Lambda}\left[\begin{array}{cc}
1 / \sin ^{2} \theta & 0 \\
0 & 1
\end{array}\right],
$$

with

$$
\bar{\Lambda}=\frac{\int d r\left(r^{4} / L(r)\right) e^{-U(r) / k_{B} T}}{\int r^{2} e^{-U(r) / k_{B} T}} .
$$

The effective dynamic equations for $\phi$ and $\theta$ can be expressed as follows:

$$
\begin{aligned}
& \frac{d \phi(t)}{d t}=\frac{\sqrt{2 k_{B} T \bar{\Lambda}}}{\sin \theta} w_{2}^{(\mathrm{S})}(t), \\
& \frac{d \theta(t)}{d t}=-\bar{\Lambda}\left(-\frac{k_{B} T}{\tan \theta}\right)+\sqrt{2 k_{B} T \bar{\Lambda}} w_{3}^{(\mathrm{S})}(t) .
\end{aligned}
$$

Unlike the case of the effective dynamic equation for $r$, eqs (73) and (74) are different from eqs (64) and (65). By performing the coarse-graining, $L(r) / r^{2}$ in eqs (64) and (65) is replaced by $\bar{\Lambda}$. The factor $\bar{\Lambda}$ depends on the mobility $L(r)$ and the bond potential $U(r)$, and the characteristic relaxation time reflects the information on $L(r)$ and $U(r)$.

\subsection{Density Field of Brownian Particles}

As the last example, we consider the overdamped interacting particles. We describe the position of the $i$-th particle as $\boldsymbol{R}_{i}$. We assume that the particles interact via the pairwise interaction potential $u(r)$, and the interaction energy is given 
as $U\left(\left\{\boldsymbol{R}_{i}\right\}\right)=\sum_{i>j} u\left(\boldsymbol{R}_{i}-\boldsymbol{R}_{j}\right)$. The Langevin equation for $\boldsymbol{R}_{i}$ is

$$
\frac{d \boldsymbol{R}_{i}(t)}{d t}=-\frac{1}{\zeta} \frac{\partial U\left(\left\{\boldsymbol{R}_{i}\right\}\right)}{\partial \boldsymbol{R}_{i}}+\sqrt{\frac{2 k_{B} T}{\zeta}} w_{i}^{(\mathrm{S})}(t),
$$

where $\zeta$ is the friction coefficient, and $\boldsymbol{w}_{i}^{(\mathrm{S})}(t)$ is the Gaussian white noise which satisfies the fluctuation-dissipation relation:

$$
\left\langle\boldsymbol{w}_{i}^{(\mathrm{S})}(t)\right\rangle=0, \quad\left\langle\boldsymbol{w}_{i}^{(\mathrm{S})}(t) \boldsymbol{w}_{j}^{(\mathrm{S})}\left(t^{\prime}\right)\right\rangle=\delta_{i j} \mathbf{1} \delta\left(t-t^{\prime}\right) .
$$

To study the dynamics of this system, the dynamic equation for the density field is sometimes useful. The (microscopic) density field is defined as

$$
\hat{\rho}(\boldsymbol{r}, t) \equiv \sum_{i} \delta\left(\boldsymbol{r}-\boldsymbol{R}_{i}(t)\right)
$$

Dean $^{41)}$ derived the dynamic equation for the density field starting from the Langevin equation (75), by using the Ito calculus. Also, we can calculate the mobility tensor for the density field by the transform in eq (27). Here we construct the dynamic equation in another way. The dissipative heat flow is calculated from eq (75) as

$$
\dot{Q}_{d}\left(\left\{\boldsymbol{R}_{i}\right\},\left\{\frac{d \boldsymbol{R}_{i}}{d t}\right\}\right)=-\sum_{i} \zeta\left(\frac{d \boldsymbol{R}_{i}(t)}{d t}\right)^{2}
$$

From eq (77), we have

$$
\frac{\partial \hat{\rho}(\boldsymbol{r}, t)}{\partial t}=J_{i \alpha}(\boldsymbol{r}) \frac{d R_{i \alpha}(t)}{d t}
$$

where $R_{i \alpha}$ represents the $\alpha$-th component of the vector $\boldsymbol{R}_{i}$, and the summation is taken for $i$ and $\alpha$. (The Einstein convention is applied.) We have defined

$$
\boldsymbol{J}_{i}(\boldsymbol{r}) \equiv-\frac{\partial}{\partial \boldsymbol{r}} \delta\left(\boldsymbol{r}-\boldsymbol{R}_{i}(t)\right)
$$

and interpret this $J_{i \alpha}(\boldsymbol{r})$ as a rectangular matrix (the dimensions for $i \alpha$ and $\boldsymbol{r}$ are different). We introduce the Moore-Penrose pseudo inverse ${ }^{42,43)} J_{i \alpha}^{+}(\boldsymbol{r})$ via: $\int d \boldsymbol{r}^{\prime} J_{j \beta}(\boldsymbol{r}) J_{j \beta}^{+}\left(\boldsymbol{r}^{\prime}\right) J_{i \alpha}\left(\boldsymbol{r}^{\prime}\right)=J_{i \alpha}(\boldsymbol{r})$. Then eq (79) can be inverted as

$$
\frac{d R_{i \alpha}(t)}{d t}=\int d \boldsymbol{r} J_{i \alpha}^{+}(\boldsymbol{r}, t) \frac{\partial \hat{\rho}(\boldsymbol{r}, t)}{\partial t}
$$

Here it would be fair to mention that eq (81) is not a unique solution of eq (79). The Moore-Penrose pseudo inverse gives the most reasonable solution and there can be other solutions.
Here we simply employ eq (81) as the solution. From eqs (78) and (81), the dissipative heat flow can be rewritten as

$$
\begin{aligned}
& \dot{Q}_{d}\left[\hat{\rho}, \frac{\partial \hat{\rho}}{\partial t}\right] \\
& =-\int d \boldsymbol{r} d \boldsymbol{r}^{\prime} \zeta J_{i \alpha}^{+}(\boldsymbol{r}) J_{i \alpha}^{+}\left(\boldsymbol{r}^{\prime}\right) \frac{\partial \hat{\rho}(\boldsymbol{r}, t)}{\partial t} \frac{\partial \hat{\rho}\left(\boldsymbol{r}^{\prime}, t\right)}{\partial t}
\end{aligned}
$$

From eq (82), the inverse mobility for the density field becomes

$$
L^{-1}\left(\boldsymbol{r}, \boldsymbol{r}^{\prime}\right)=\zeta J_{i \alpha}^{+}(\boldsymbol{r}) J_{i \alpha}^{+}\left(\boldsymbol{r}^{\prime}\right)=\zeta\left[J_{i \alpha}(\boldsymbol{r}) J_{i \alpha}\left(\boldsymbol{r}^{\prime}\right)\right]^{-1}
$$

Here we utilize the explicit expression for the Moore-Penrose pseudo inverse: $J_{i \alpha}^{+}(\boldsymbol{r})=\int d \boldsymbol{r}^{\prime} J_{i \alpha}\left(\boldsymbol{r}^{\prime}\right)\left[J_{j \beta}\left(\boldsymbol{r}^{\prime}\right) J_{j \beta}(\boldsymbol{r})\right]^{-1}$. Therefore, we have the following simple expression for the mobility tensor:

$$
\begin{aligned}
L\left(\boldsymbol{r}, \boldsymbol{r}^{\prime}\right) & =\frac{1}{\zeta} J_{i \alpha}(\boldsymbol{r}) J_{i \alpha}\left(\boldsymbol{r}^{\prime}\right) \\
& =\frac{1}{\zeta} \sum_{i} \frac{\partial}{\partial \boldsymbol{r}} \delta\left(\boldsymbol{r}-\boldsymbol{R}_{i}\right) \cdot \frac{\partial}{\partial \boldsymbol{r}^{\prime}} \delta\left(\boldsymbol{r}^{\prime}-\boldsymbol{R}_{i}\right) \\
& =\frac{\partial}{\partial \boldsymbol{r}} \cdot\left[\frac{\hat{\rho}(\boldsymbol{r})}{\zeta} \frac{\partial}{\partial \boldsymbol{r}^{\prime}} \delta\left(\boldsymbol{r}-\boldsymbol{r}^{\prime}\right)\right] .
\end{aligned}
$$

From our derivation, eq (84) may not be exact, but it works as the most reasonable expression, which would be a good approximation in many cases. (Fortunately, the exact derivation by Dean ${ }^{41)}$ gives the same mobility tensor and thus our method works perfectly for this system.) By combining the mobility tensor (84) with the free energy for the density field ${ }^{41,44)}$

$$
\begin{aligned}
\mathcal{F}[\hat{\rho}]= & k_{B} T \int d \boldsymbol{r} \hat{\rho}(\boldsymbol{r})[\ln \hat{\rho}(\boldsymbol{r})-1] \\
& +\frac{1}{2} \int d \boldsymbol{r} d \boldsymbol{r}^{\prime} \hat{\rho}(\boldsymbol{r}) u\left(\boldsymbol{r}-\boldsymbol{r}^{\prime}\right) \hat{\rho}\left(\boldsymbol{r}^{\prime}\right),
\end{aligned}
$$

we can construct the Langevin equation for the density field. For convenience, we introduce the following noise coefficient tensor

$$
\boldsymbol{B}\left(\boldsymbol{r}, \boldsymbol{r}^{\prime}\right)=-\sqrt{\frac{\hat{\rho}\left(\boldsymbol{r}^{\prime}\right)}{\zeta}} \frac{\partial}{\partial \boldsymbol{r}^{\prime}} \delta\left(\boldsymbol{r}-\boldsymbol{r}^{\prime}\right)
$$

which satisfies $\int d \boldsymbol{r}^{\prime} \boldsymbol{B}\left(\boldsymbol{r}, \boldsymbol{r}^{\prime}\right) \cdot \boldsymbol{B}\left(\boldsymbol{r}^{\prime \prime}, \boldsymbol{r}^{\prime}\right)=L\left(\boldsymbol{r}, \boldsymbol{r}^{\prime \prime}\right)$. Then the Ito type Langevin equation becomes as follows, which is equivalent to the Dean's equation: ${ }^{41)}$ 


$$
\begin{aligned}
\frac{\partial \hat{\rho}(\boldsymbol{r}, t)}{\partial t}= & \int d \boldsymbol{r}^{\prime}\left[-L\left(\boldsymbol{r}, \boldsymbol{r}^{\prime}\right) \frac{\delta \mathcal{F}[\hat{\rho}]}{\delta \hat{\rho}\left(\boldsymbol{r}^{\prime}, t\right)}+k_{B} T \frac{\delta L\left(\boldsymbol{r}, \boldsymbol{r}^{\prime}\right)}{\delta \hat{\rho}\left(\boldsymbol{r}^{\prime}, t\right)}\right. \\
& \left.+\sqrt{2 k_{B} T} \boldsymbol{B}\left(\boldsymbol{r}, \boldsymbol{r}^{\prime}\right) \cdot \boldsymbol{w}^{(\mathrm{I})}\left(\boldsymbol{r}^{\prime}, t\right)\right] \\
= & \frac{\partial}{\partial \boldsymbol{r}} \cdot\left[\frac{\hat{\rho}(\boldsymbol{r}, t)}{\zeta} \frac{\partial}{\partial \boldsymbol{r}} \frac{\delta \mathcal{F}[\hat{\rho}]}{\delta \hat{\rho}(\boldsymbol{r}, t)}\right] \\
& +\frac{\partial}{\partial \boldsymbol{r}} \cdot\left[\sqrt{\frac{2 k_{B} T \hat{\rho}(\boldsymbol{r}, t)}{\zeta}} \boldsymbol{w}^{(\mathrm{I})}(\boldsymbol{r}, t)\right],
\end{aligned}
$$

where $\boldsymbol{w}^{(\mathrm{I})}(\boldsymbol{r}, t)$ is the Gaussian white noise field which satisfies

$$
\begin{aligned}
& \left\langle\boldsymbol{w}^{(\mathrm{I})}(\boldsymbol{r}, t)\right\rangle=0, \\
& \left\langle\boldsymbol{w}^{(\mathrm{I})}(\boldsymbol{r}, t) \boldsymbol{w}^{(\mathrm{I})}\left(\boldsymbol{r}^{\prime}, t^{\prime}\right)\right\rangle=\mathbf{1} \delta\left(\boldsymbol{r}-\boldsymbol{r}^{\prime}\right) \delta\left(t-t^{\prime}\right) .
\end{aligned}
$$

If we ignore the stochastic term in eq (87), it reduces to the diffusion type equation under the mean-field approximation.

As Dean showed, eq (87) can be directly obtained from the Langevin equation (75) in as straightforward way. The Langevin equation for the density field itself is not a new result. Moreover, the original derivation by Dean is simple and does not need to handle the mobility tensor explicitly. Nonetheless our method will be useful because it provides an alternative way to construct the Langevin equation. The interpretation of the Dean's equation is not that simple, and various studies have been done. ${ }^{45-48)}$ In the Dean's original derivation, the Langevin equation for the density field is directly derived from the Langevin equation for particles (eq (75)). The derivation itself is mathematically straightforward, but the physical interpretation of the microscopic density field and how to perform the coarse-graining is not that clear. ${ }^{47,48)}$ In the thus obtained dynamic equation, the mobility tensor and the free energy are mixed up and one should separate them manually. On the other hand, our method directly gives the expression of the mobility tensor (although the calculation is not simple). The free energy is obtained from the partition function, and this calculation is independent of the Langevin equation. Also, our method can be applied for the coarse-graining (at least in principle). We expect that our method will provide a new aspect when we study the Dean's and related dynamic equations.

Doi $^{27)}$ claimed that a similar diffusion type equation can be obtained by the Onsager's method. However, in the Doi's derivation, the Rayleigh dissipation function is expressed in terms of the particle velocity field $\hat{\boldsymbol{v}}(\boldsymbol{r}, t)$. The velocity field is defined via

$$
\frac{\partial \hat{\rho}(\boldsymbol{r}, t)}{\partial t}=-\frac{\partial}{\partial \boldsymbol{r}} \cdot[\hat{\rho}(\boldsymbol{r}, t) \hat{\boldsymbol{v}}(\boldsymbol{r}, t)]
$$

and the Rayleigh's dissipation function is assumed to be

$$
\Psi=\frac{1}{2} \int d \boldsymbol{r} \zeta \hat{\rho}(\boldsymbol{r}) \hat{\boldsymbol{v}}^{2}(\boldsymbol{r})
$$

Then, Doi constructed the Rayleighian from eq (90) and the free energy (85) and derived the dynamic equation which is similar to eq (87) (without the noise term) by minimizing the Rayleighian with respect to $\hat{\boldsymbol{v}}(\boldsymbol{r})$. However, this derivation lacks the consistency. The Rayleigh's dissipation function (which is essentially the same as the dissipative heat, from eq (19)) should be interpreted as a functional of the slow variable $\hat{\rho}(\boldsymbol{r})$ and its time derivative $\partial \hat{\rho}(\boldsymbol{r}) / \partial t$. To be consistent, the minimization should be performed for $\partial \hat{\rho}(\boldsymbol{r}) / \partial t$, not for other variables such as $\hat{v}(\boldsymbol{r})$. (Doi ${ }^{28)}$ also showed a similar minimization with respect to another variable $\hat{\boldsymbol{j}}(\boldsymbol{r}) \equiv \hat{\rho}(\boldsymbol{r}) \hat{\boldsymbol{v}}(\boldsymbol{r})$.) On the other hand, in our derivation, the dissipation function (the dissipative heat flow) is interpreted as a functional of $\hat{\rho}(\boldsymbol{r})$ and $\partial \hat{\rho}(\boldsymbol{r}) / \partial t$, and thus is systematic and fully consistent with the general method. (Doi's derivation gives the correct dynamic equation because the variable $\hat{\boldsymbol{v}}(\boldsymbol{r})$ is linear in $\partial \hat{\rho}(\boldsymbol{r}) / \partial t$. If the variable for the minimization is not linear in $\partial \hat{\rho}(\boldsymbol{r}) / \partial t$, of course, the resulting dynamic equation becomes incorrect.)

\section{CONCLUSIONS}

We analyzed the dissipation of the mesoscopic systems which obey the Langevin equation, from the view point of the stochastic energetics. From the energy balance equation, we obtained the dissipative and fluctuating heat flows. The dissipative heat flow has a similar form to the Rayleigh dissipation function, but the physical meaning is much clearer; the dissipative heat flow simply represents the heat which moves from the bath to the system in absence of the fluctuation.

We showed that the dissipative heat flow can be used to calculate the (inverse) mobility. The dissipative heat flow can be related to the mobility tensor, and in fact, we can systematically calculate the mobility tensor once the dissipative heat flow is obtained. We also showed that the dissipative heat flow is in a covariant form, whereas the free energy is not in a covariant form. (This means that, neither our energy balance equation nor the Rayleighian is covariant.) To construct the Langevin equation for a specific variable, we can utilize the dissipative heat flow calculated for other variables. Moreover, the dissipative heat flow can be utilized to perform the coarsegraining. The mobility tensor of the coarse-grained variables can be systematically constructed by calculating the approximate dissipative heat flow in local equilibrium. Then we can 
construct the Langevin equation by combining the free energy and the obtained mobility tensor. Our method is similar to but qualitatively different from the Reyleigh's and Onsager's methods, and can be utilized as an alternative to them.

As examples, we applied our method to several systems such as the dumbbell model and the Brownian particles. Our method can successfully reproduce the Dean's equation for the density field of the interacting Brownian particles. Our method gives the dynamic equations and coarse-grained effective dynamic equations for these systems which are consistent with those obtained by the conventional methods. We consider that our method will be useful to construct the dynamic equation models to study rheological properties of some soft matter systems. Of course, the usefulness strongly depends on the properties of the target system. Judging from the results in this work, we conclude that there is no general construction method for mesoscopic and macroscopic dynamic equations, which is variational and covariant. Our method may be convenient for some cases, yet the conventional methods without the dissipative heat flow (and the Rayleigh dissipation function) may be more convenient for some other cases. We should emphasize that our method (or any other method) is never a "silver bullet." Our method is just one candidate among various methods, and one should carefully choose an appropriate method to construct dynamic equations.

\section{ACKNOWLEDGMENT}

The author thanks Prof. M. Doi (Beihang University) for comments and discussions. This work was supported by JST, PRESTO Grant Number JPMJPR1992, Japan, and Grant-inAid (KAKENHI) for Scientific Research B JP19H01861.

\section{REFERENCES}

1) Larson RG, "The Structure and Rheology of Complex Fluids", (1999), Oxford University Press, New York.

2) Sekimoto K, "Stochastic Energetics”, (2010), Springer, Berlin.

3) van Kampen NG, "Stochastic Processes in Physics and Chemistry”, 3rd ed, (2007), Elsevier, Amsterdam.

4) Gardiner CW, "Handbook of Stochastic Methods", 3rd ed, (2004), Springer, Berlin.

5) Öttinger HC, "Stochastic Processes in Polymeric Fluids: Tools and Examples for Developing Simulation Algorithms”, (1996), Springer, Berlin.

6) Onuki A, “Phase Transition Dynamics”, (2002), Cambridge University Press, Cambridge.

7) Grmela M, Öttinger HC, Phys Rev E, 56, 6620 (1997).

8) Öttinger HC, Grmela M, Phys Rev E, 56, 6633 (1997).
9) Landau LD, Lifshitz EM, Mechanics, 2nd ed, (1969), Pergamon Press, Oxford.

10) Lanczos C, “The Variatonal Principles of Mechanics”, (1949), University of Toronto Press, Toronto.

11) Strutt JW, Proc London Math Soc, s1-4 (1871).

12) Strutt JW, “The Theory of Sound”, (1877), Cambridge University Press, Cambridge.

13) Bateman H, Phys Rev, 38, 815 (1931).

14) Riewe F, Phys Rev E, 55, 3581 (1997).

15) Lazo MJ, Krumreich CE, J Math Phys, 55, 122902 (2014).

16) Galley CR, Phys Rev Lett, 110, 174301 (2013).

17) Glavatskiy KS, J Chem Phys, 143, 164101 (2015).

18) Martinez-Perez NE, Ramirez C, J Math Phys, 59, 032904 (2018).

19) Minguzzi E, Eur J Phys, 36, 035014 (2015).

20) Minguzzi E, J Geom Mech, 7, 473 (2015).

21) Allison A, Pearce CEM, Abbott D, PLOS One, 9, e77190 (2017).

22) Suzuki M, Physica A, 392, 314 (2013).

23) Onsager L, Phys Rev, 37, 405 (1931).

24) Onsager L, Phys Rev, 38, 2265 (1931).

25) Onsager L, Machlup S, Phys Rev, 91, 1505 (1953).

26) Bauer PS, Proc Nat Acad Sci, 17, 311 (1931).

27) Doi M, “Soft Matter Physics”, (2013), Oxford University Press, Oxford.

28) Doi M, Zhou J, Di Y, Xu X, Phys Rev E, 99, 063303 (2019).

29) Itami M, Sasa S, J Stat Phys, 167, 46 (2017).

30) Evans DJ, Morris GP, "Statistical Mechanics of Nonequilibrium Liquids”, 2nd ed, (2008), Cambridge University Press, Cambridge.

31) Risken H, “The Fokker-Planck Equation”, 2nd ed, (1989), Springer, Berlin.

32) Jeon JH, Chechkin AV, Metzler R, Phys Chem Chem Phys, 16, 15811 (2014)

33) Uneyama T, Miyaguchi T, Akimoto T, Phys Rev E, 92, 032140 (2015).

34) Uneyama T, Miyaguchi T, Akimoto T, Phys Rev E, 99, 032127 (2019).

35) Sekimoto K, Phys Rev E, 76, 060103 (2007).

36) Pauli W, “Theory of Relativity”, (1958), Pergamon Press, New York.

37) Nakamura T, arXiv:1803.09034, (2018).

38) Kawasaki K, J Phys A Math Nucl Gen, 6, 1289 (1973).

39) Uneyama T, Nakai F, Masubuchi Y, Nihon Reoroji Gakkaishi (J Soc Rheol Jpn), 47, 143 (2019).

40) Neumann RM, Am J Phys, 48, 354 (1980).

41) Dean DS, J Phys A Math Gen, 29, L613 (1996).

42) Ben-Israel A, Greville TNE, “Generalized Inverses: Theory and Applications”, (1977), Wiley, New York.

43) Petersen KB, Pedersen MS, “The Matrix Cookbook", ver 20121115, (2012), http://www2.imm.dtu.dk/pubdb/views/ publication_details.php?id=3274. 
44) Hansen JP, McDonald IR, “Theory of Simple Liquids", 3rd ed, (2006), Elsevier, Amsterdam.

45) Marconi UMB, Tarazona P, J Chem Phys, 110, 8032 (1999).

46) Frusawa H, Hayakawa R, J Phys A Math Gen, 33, L155 (2000).
47) Archer AJ, Rauscher M, J Phys A Math Gen, 37, 9325 (2004).

48) Duran-Olivencia MA, Yatsyshin P, Goddard BD, Kalliadasis S, New J Phys, 19, 123022 (2018). 